occur. The interesting thing is that all these abnormalities can return to normal if the patient loses the excess weight. Hypoventilation Syndrome:

1. Pickwickian syndrome. This is very similar to the pattern produced by gross obesity and, in fact, gross obesity is one of the characteristics of this syndrome. In addition there is marked somnilence, cyanosis and polycythaemia. The syndrome derives its name, of course, from the fat boy of 'The Pickwick Papers' (Charles Dickens).

2. Damage to the respiratory centre. This may occur as a result of encephalitis and these patients may present with no pulmonary disability but with polycythaemia and cyanosis. Once again, the characteristic finding is that of alveolar hypoventilation.

Polycythaemia: These patients are frequently brought for pulmonary function tests with a view to deciding whether this is a primary deviation of the bone marrow or secondary to pulmonary pathology. For the most part, arterial oxygen levels are normal in primary polycythaemia and considerably reduced in polycythaemia due to pulmonary pathology. A full range of pulmonary function tests frequently provides the answer in this type of problem.

Assessment of Progress.

The degree of bronchospasm is easily estimated using some form of timed vital capacity or maximum breathing capacity and then comparing the results before and after a broncho-dilator drug. Periodic estimations also give a very good idea of long term therapy.

\section{Chronic Bronchitis and Emphysema}

$B y$ Dr. L. D. ERASMUS, M.B., B.Ch.(Rand)M.D. (Pretoria) Member of the Faculty of Medicine, Pretoria University.

A Lecture given to Delegates and Members of the South African Society of Physiotherapy on the first day of the "Chest Course", which was held at the Pretoria Hospital Physiotherapy School on May 27th and 28th, 1960.

Although either of these conditions may occur separately they are so frequently associated as almost to appear inseparable. An allergic factor or frank bronchial asthma is a frequent accompaniment. Chronic bronchitis and emphysema are conditions of paramount importance because of the high morbidity and even mortality brought about by acute exacerbations and complications. They occur frequently and result in tens of thousands of lost man-hours every year and treatment is frequently expensive, drawn out and usually requires hospitalization.

Clinical History.

Established chronic bronchitis and emphysema is usually preceded for several years by chronic cough which is usually productive and for several years before the pattern becomes established frequent attacks of acute winter bronchitis occur. Attacks of asthma are not infrequent. Later persistent exertional dyspnoea occurs and this may be interspersed with more acute attacks of bronchospasm.

The attacks of bronchitis may be suppurative in nature due to severe secondary infection and especially in the elderly disabling and even fatal attacks of broncho-pneumonia may occur. These attacks frequently precipitate congestive cardiac failure which also occurs particularly in the older age group when arteriosclerosis and hypertension have become established and have added another burden. Secondary polycythaemia frequently increases the blood viscosity and occasionally papiloedema and blindness may follow.

Aetiology.

External factors are frequently of the greatest importance and the incidence of chronic bronchitis is highest in those areas where there is pollution of the atmosphere by industrial gases and dust. Chronic bronchitis is also more frequent in heavy smokers. Pollution of the atmosphere is becoming a world wide problem. We breathe as much as $3 \pm$ Ibs. of air by weight in 24 hours, equal in weight to tin food we consume, but for many years it has not occurred to
Emphysema is usually permanent and frequently progressive and it is frequently only associated bronchospasm or infection that can be influenced by treatment. Pulmonary function tests have been studied before and after the application of physiotherapy to these patients and it appears that the degree of pulmonary function deviation is not influenced by physiotherapy.

Patients with pleurisy and pleural effusion show considerable improvement in their vital capacity as the condition improves.

Congestive cardiac failure. The degree of pulmonary oedema is indicated by a reduction in the vital capacity. In some instances pulmonary congestion can be determined at an early stage by measuring the vital capacity.

The progress of patients with weakness of the respiratory muscles can sometimes be effectively followed by doing the maximum breathing capacity test. This is repeated at short intervals and fatiguability will be indicated by progressive reduction in subsequent tests. A similar cluster of tests can be done at an interval of days or even weeks and progress followed in this fashion.

In conclusion it may be said that pulmonary function tests do not replace other methods of assessing the condition of the patient's lungs but they are in many instances a very valuable adjunct.

men to be as fastidious about the air they breathe as the food they eat. We will probably have to put up with grossly contaminated atmospheres for many years to come. Irritation from dust in dusty occupations such as mining is also of considerable importance.

An infective agency is important and the commonest infecting organism is $\mathbf{H}$. influenza but a mixed bacterial flora is frequently found. Individual predisposing factors include a hereditary tendency and allergy.

Basic Pathology.

It is convenient to consider the respiratory unit as consisting of a terminal and a respiratory bronchiole leading to the alveolar sacs. This may be represented in the following fashion in the normal
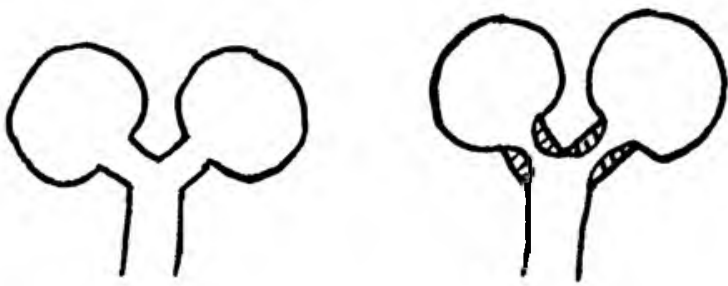

and thus when there is bronchial inflamation or spasm i.e., with an obstructive factor introduced.

With emphysematous dilation, rupture and coalescence of alveolar sacs occur as the result of degenerative changes in the elastic fibres of the alveolar walls. The factor of bronchial obstruction and infection is frequently superadded and there may be associated inter-alveolar fibrosis. These changes result in:-

1. A mechanical ventilatory deficiency where expiration especially is difficult due to bronchiolar obstruction, loss of elastic tissue and the patient having to expire from an initial level of hyperinflation.

2. Inefficient distribution of the air within the alveolar sacs occurs and the impaired diffusion of the gasses occurs.

3. In turn anoxaemia and carbon dioxide accumulation occurs in the blood resulting in acidosis and in compensatory increase of the blood bicarbonate.

In terms of pulmonary function tests the lung volume may remain the same (as will the vital capacity in many instances) but expiratory difficulty results in air trapping, 


\section{ASPECTS OF RESPIRATION (Continued from Page4).}

(iii) Strength (size) of the respiratory muscles-inspiratory and expiratory (cf. the respiratory muscles of the trained athlete);

(iv) Elasticity of the lungs (cf. the reduced vital capacity in all forms of fibrosis of the lungs);

(v) Functional efficiency of the nervous structures concerned:

(a) respiratory centre,

(b) anterior motor neurons (cf. poliomyelitis),

(c) peripheral efferent or motor nerves to the respiratory muscles.

\section{Why do the inspiratory muscles relax?}

Because they receive no impulses from the spinal cord motor neurons, which in turn receive no impulses from the inspiratory centre. Thus, the continuous discharge of repetitive impulses by the inspiratory neurons has temporarily been stopped or inhibited, or inspiration has been cut short to allow expiration to take place.

The responsible mechanisms for this are:

(i) pneumotaxic centre,

(ii) expiratory centre,

(iii) Hering-Breuer reflex (vagal mechanism).

\section{Pneumotaxic Centre.}

This consists of respiratory neurons scattered in the formatio reticularis of the pons. They make synaptical contact with those of the inspiratory and expiratory centres.

As the inspiratory neurons discharge to the anterior motor neurons, there is a simultaneous discharge to the pneumotaxic neurons. These in turn discharge to the expiratory neurons. At a certain critical level of excitation, the latter emit a barrage of impulses at the inspiratory neurons, which for a short period, completely inhibits their discharge.

\section{Hering-Breuer reflex.}

The delicate alveolar membranes of the lung contain receptors sensitive to the amount of stretch of inflation of the lung-stretch receptors.

As a lung becomes progressively more inflated during inspiration, these receptors are stimulated and a stream of afferent impulses is conducted along afferent vagus fibres to the expiratory neurons, which again inhibit the inspiratory neurons, allowing expiration to take place.

The rhythm of breathing is therefore the result of the inter action of the inspiratory, expiratory and pneumotoxic centres and the vagal mechanism.

Whereas the depth of an inspiration is determined by the degree of discharge by the inspiratory neurons the rate of respiration is dependent on the activity of the pneumotaxic and vagal mechanisms.

\section{How is respiration regulated?}

The respiratory pattern (i.e. depth and rate) is regulated, via the respiratory centre, by:

(i) chemical substances, and

(ii) nerve impulses.

\section{Chemical Substances.}

Under all conditions of normal, eupneic breathing, the respiratory pattern is regulated almost exclusively by the $\mathrm{CO}_{2}$ content of the Arterial blood. This is mediated through a direct action of the $\mathrm{HCO}_{3}$ (bicarbonate) ions on the respiratory neurons and changes in acidity $(\mathrm{pH})$ caused by increased or decreased $\mathrm{CO}_{2}$.
The normal tension of $\mathrm{CO}_{2}\left(\mathrm{pCO}_{2}\right)$ in arterial blood $=$ $40 \mathrm{~mm}$. Hg. Increasing this volume by as little as $2 \mathrm{~mm}$. $\mathrm{Hg}$. is sufficient to double pulmonary ventilation (hyperpnoea); hence the hyperpnoea of acidosis. Similarly a slighi decrease of this value causes apnoea; hence the slow shallow breathing of alkalosis.

In certain cases of anoxia, the respiratory pattern is controlled by the $\mathrm{O}_{2}$ content $\left(\mathrm{pO}_{2}\right)$ of the arterial blood, via the chemoreceptors of the aortic and carotid bodies.

\section{Nerve Impulses.}

These have already been referred to elsewhere.

The hyperpnoea of muscular activity is largely the effect of afferent impulses from the contracting muscles and moving joints of the respiratory centre.

During other physiological activities, e.g. talking, sneezing, coughing, swallowing, etc., the basic respiratory pattern (as controlled by the $\mathrm{CO}_{2}$-content of the arterial blood) is modified by nerve impulses from the respective nerve centres, acting on the neurons of the respiratory centre.

\section{PULMONARY TEST (Continlted from Page 7).}

reduction of timed vital capacity and the maximum breathing capacity. Residual volume is usually considerably increased both as an absolute value and as a proportion of the total lung capacity.

\section{Clinical Features.}

An advanced cause of emphysema will then be seen to have anoxaemia with cyanosis heightened frequently by secondary polycythaemia and shows the considerable ventilatory difficulty associated with hyperinflation of the lungs and diffuse expiratory obstruction. Clinically the patient wheezes on the slightest exertion or even at rest and has to use his accessory muscles of respiration. The chest becomes barrel-shaped and the ribs horizontally placed. Respiration is carried out partially by diaphragmatic excursion and "en bloc" movement of the barrel-shaped thoracic cage. On ausculation the prolonged expiratory difficulty of a forced maximal expiration is usually quite easily detected and in my opinion this is the most valuable single sign in the clinical assessment of the patient. For the most part also there is reduction in air entry as judged by the stethoscope.

\section{Treatment.}

Treatment is difficult and prolonged in most instances. It has to be said at the outset that emphysema as such cannot be influenced by treatment and any therapeutic attack is directed against bronchospasm, infection and viscid secretions with a variety of agents including antibiotics, antispasmodics, steroids, detergent and wetting inhalant and enzymes (streptokinase and trypsin).

Although there is not the slightest doubt in my mind that Physiotherapy is of considerable value in making more efficient use of the patient's natural capacity for ventilation, there is no objective improvement in pulmonary function tests in controlled series of patients in whom this has been measured before and after physiotherapy.

Complications (especially bronchopneumonia and corpulmonale) are of a serious nature and require vigorous treatment in their own right. Frequently then we have a patient who is very ill with grossly reduced respiratory reserve and considerable secondary infection in the lungs with associated cardiac failure and a wide, vigorous and persistent therapeutic attack is indicated. As far as future developments are concerned, however, prevention is likely to prove more rewarding than an attempt at cure. 